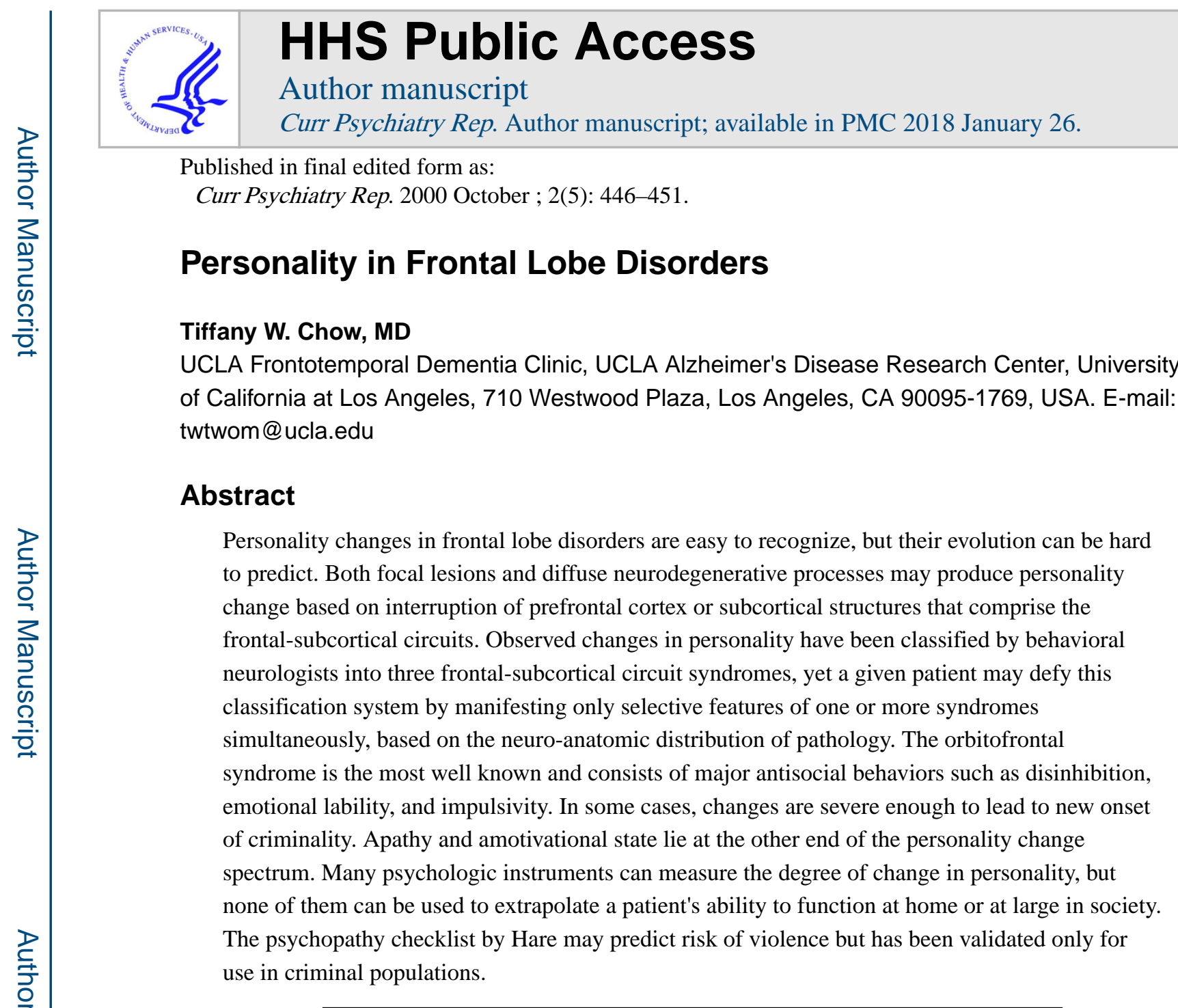

\title{
Introduction
}

This review uses the Diagnostic and Statistical Manual of Mental Disorders fourth edition (DSM-IV) definition of personality as "enduring patterns of perceiving, relating to, and thinking about the environment and self that are exhibited in a wide range of social and personal contexts" [1]. Emotion, behavior, and cognition converge in personality. Impairment of any of these three cornerstones can create a marked change in personality (Fig. 1). For example, depression in a previously independent leader can influence cognition and behavior to an amotivational state and relatively introverted behavior to create a reclusive personality type. Personality changes in this review refer to those social and personal interactions that become dysfunctional as a direct result of pathology in the frontal lobes. Premorbid personality types and their associated risk of head trauma or dementia are not discussed.

The altered patterns of social and personal interactions in patients with frontal lobe disorders are often generalized as the "frontal lobe personality," in which disinhibition and impulsivity dominate. The transformation of Phineas Gage from a mild-mannered, responsible foreman to an aggravated, antisocial deviant after obliteration of the left frontal lobe is notorious among lay people $[2,3]$. However, personality changes after onset of frontal lobe disorders 
have heterogeneous presentations and may instead exhibit themselves in apathy and abulia or alternating features of apathy and agitation. The frontal lobes comprise a large proportion of the human brain, and focal lesions within the frontal lobes elicit different manifestations of behavior. Neurodegenerative processes affect the brain more diffusely than neoplasm or stroke. When dementing illnesses involve the frontal lobes, it is more difficult to predict exactly which personality changes will occur or how the natural history of the personality change will progress. This review 1) describes named syndromes of personality change that have been observed in frontal lobe disorders, 2) lists personality changes that have been strongly associated with specific frontal lobe disorders, and 3) discusses instruments for assessment of personality in these contexts.

\section{Fronto-subcortical Circuits}

In addition to its contributions to motor and oculomotor function, the frontal lobe has three cortical-subcortical circuits that subserve mood, cognition, and behavior [4]. Lesions at either cortical or subcortical stations along each circuit can result in well-characterized, circuit-specific or circuit-related clinical syndromes (Fig. 2).

\section{Orbitofrontal Syndrome}

Features of the orbitofrontal syndrome, also known as Acquired Sociopathy after Tranel and Damasio, appear in Table 1. This syndrome most closely approximates the "frontal lobe personality" assumed by many to occur after injury to the frontal lobes. Tranel [5] and Damasio et al. [2] studied the localization of stroke, head trauma, or neoplasm-induced lesions in patients with acquired sociopathy. Six of nine patients with this clinical syndrome had bilateral orbitofrontal cortical damage, two of the nine bore a right-sided lesion and the last had a left-sided lesion. Fearlessness and disregard for emotional outcomes may be associated with the dysregulated physiologic response to emotional stimuli observed in patients with frontal lobe lesions [6].

\section{Anterior Cingulate and Dorsolateral Syndromes}

As often as orbitofrontal syndromes occur, many patients with frontal lobe disorders instead manifest either an anterior cingulate syndrome or dorsolateral prefrontal cortex syndrome $[4,7 \bullet \bullet, 8]$. Patients with the anterior cingulate syndrome tend to become withdrawn, quiet, apathetic, and sometimes abulic, which contrasts starkly against changes of the orbitofrontal syndrome. Patients with dorsolateral prefrontal cortex syndromes are less organized in their approach to environment and lack the ability to plan. They do not use appropriate judgment based on these disabilities and may seem relatively disinhibited while maintaining social skills.

\section{Predicting Clinical Syndromes from Neuroanatomic Localization}

Even small lesions in subcortical areas such as caudate, globus pallidus, or thalamus often disrupt more than one frontal-subcortical circuit due to the close proximity of the circuits in these areas [8]. Rare patients with clearly lesioned frontal lobes do not develop the ascribed behavioral or cognitive changes. In one study, one of 53 patients with orbitofrontal cortical 
lesions had no features of the clinical syndrome in Table 1 [5]. Thus it can be difficult to find a patient with a classic, predictable syndrome in the course of everyday practice. The majority of patients with frontal lobe disorders due to decelerated closed head injury have orbitofrontal damage $[9 \bullet \bullet]$.

\section{Cortical Injury}

One can predict personality changes from focal lesions, if the neuroanatomy is quite clearly confined to one area of prefrontal cortex (Table 2). Stroke, neoplasm, or well-circumscribed focal infectious processes such as bacterial abscess formation may cause an orbitofrontal, anterior cingulate, or dorsolateral prefrontal cortical-subcortical syndrome if they involve the appropriate cortical regions. At times, resection of a slow-growing tumor in the frontal lobe results in marked alteration of personality. Depressive versus manic mood changes have not consistently associated with laterality of the lesion sustained [10]. A large vessel middle cerebral artery stroke may result in a combination of orbitofrontal and dorsolateral syndromes, whereas a stroke in the distribution of both anterior cerebral arteries may cause the abulic phenomena of the anterior cingulate syndrome.

\section{Subcortical Lesions}

Lesions in basal ganglia may cause one or more of the frontal-subcortical syndromes by dint of the convergence of all three circuits as they pass through these areas. Tourette's syndrome and obsessive-compulsive disorder are accompanied by abnormal activity in basal ganglia structures and prefrontal cortex on functional neuroimaging [11,12]. Whether these areas are hyper- or hypoactive has not been firmly established, but acquired Tourette's syndrome and obsessive-compulsive disorder have been observed in pediatric autoimmune neuropsychiatric disorders associated with streptococcal infections, in which antibodies against group A Streptococcus cross-react against basal ganglia.[13,14,15•]

Some neurodegenerative disorders involve basal ganglia and so result in personality changes: depression and negativity dominate the personality changes observed in Parkinson's disease, Huntington's disease, and progressive supranuclear palsy, but anxiety, compulsiveness, and restlessness have also been described [7]. In these cases, treatment for movement disorders may be an iatrogenic, reversible etiology for this type of personality change. Corticobasal ganglionic degeneration has an asymmetric predilection for basal ganglia and parietal more than frontal cortex. Patients often present to the neurologist with apraxia, asymmetric parkinsonism, and visuospatial deficits. The clinical criteria for corticobasal ganglionic degeneration do not include changes in mood or personality, but case reports note inflexible behavior, emotional lability, and depression [16,17].

\section{Dementias and Other Diffuse Processes}

Diffuse processes affecting frontal lobes may affect cortex and cortico-ortical connections.

Both cortical and subcortical dementias are common in the elderly, but personality does not ordinarily change as part of normal aging [18]. Frontal cortex is affected in both frontotemporal dementia (FTD) and Alzheimer's disease (AD), but among FTD patients, 
personality changes are much more marked and more frequently represent socially undesirable behaviors [19-22]. Disinhibition, euphoria, obsessive-compulsive behaviors, and hoarding are more frequent in FTD than AD patients [19,23].

\section{Frontotemporal dementia}

Frontotemporal dementia patients with predominantly right-sided pathology have less insight regarding their personality changes than patients with left-sided pathology [24]. The personality change includes not only poor expression of affect but also the disabled interpretation of affect in others. FTD patients are less accurate than AD patients in comprehending facial expressions and prosody [24].

\section{Alzheimer's disease}

Patients with Alzheimer's disease are frequently apathetic or depressed [19]. This may be related to changes in frontal regions that blunt autonomic adjuncts to behavior and mood. Cortical layers III and V of posterior orbitofrontal cortex and Brodmann's area 25 of ventromedial frontal cortex contain cortico-autonomic projections. AD patients with a mean 7.5-year duration of illness have similar neurofibrillary tangle densities in both these frontal areas and temporal cortex [25]. Psychophysiologic experiments have demonstrated that AD patients do not register environmental stimuli with the appropriate autonomic response [26]. Functional imaging with fluorodeoxyglucose positron emission tomography has given further in vivo evidence to support the role of frontal lobes in mood changes: the severity of depression in $\mathrm{AD}$ patients correlates with hypometabolism in bilateral superior frontal and left anterior cingulate cortex [27].

\section{Dementia with Lewy bodies}

Dementia with Lewy bodies manifests clinically as coincident parkinsonism and cognitive decline. Fluctuation in level of consciousness and deficits in attention distinguish dementia with Lewy bodies from $\mathrm{AD}$ and are thought to reflect pathologic involvement of frontalsubcortical circuits [28••]. As in $\mathrm{AD}$, these patients frequently manifest apathy, which may represent dysfunction in the anterior cingulate-subcortical circuit [29•].

\section{Trauma}

Neurodegenerative disorders are not the only diffuse processes that change personality. Traumatic brain injury and closed head injury may cause diffuse axonal injury due to shear forces on white matter tracts. This effectively disconnects cortical regions from one another and can recreate any of the frontal-subcortical syndromes [9]. Less apparent neuroanatomic changes can also result in personality change after a concussion. Elements of the postconcussion disorder, also known as persistent post-concussion syndrome, include social or sexual inappropriateness, irritability, aggression on little provocation, anxiety, depression, apathy, and emotional lability. Physical symptoms of this disorder usually include headache, vertigo, and fatigue [1]. 


\section{Epilepsy}

Epilepsy with frontal lobe foci has been associated with DSM-defined personality disorders. Lopez-Rodriguez et al. [30] did not find a significantly different frequency of cluster B (dramatic/emotional) or cluster $\mathrm{C}$ (anxious/fearful) personality disorders between 37 patients with refractory temporal lobe epilepsy and 12 patients with non-temporal lobe epilepsy ( 4 of 12 had frontal lobe seizures). Among epileptic patients, personality may be determined by multiple cofactors: dysfunction at the seizure focus, aural phenomena, reactions to the debilitating social sequelae of refractory epilepsy, and medication effects. Discussion of the temporal lobe (epilepsy) personality or Geschwind syndrome falls beyond the scope of this review.

\section{Role of Chronicity in Frontal Lobe Disorders}

In addition to localization of the lesion, timing of the insult to frontal lobes can change an individual's personality by hindering his acquisition of social and moral reasoning. Anderson et al. [31] studied two patients with frontal lobe disorder onset prior to age 2 years. Although both patients showed intact cognitive performances on standardized neuropsychologic testing, they failed to respond normally to punishment contingencies and interventions to alter behaviors, in ways that echoed their real-life behavioral problems. In contrast, patients who develop frontal lobe dysfunction due to a dementia in later adulthood are often able to respond appropriately when asked how they would react to various test stimuli but betray their changes in personality in situations outside of the clinical context.

\section{Measures of Personality}

Instruments used to assess personality in frontal disorder patients yield data on premorbid versus current changes in personality and quantitative comparisons of behavioral disturbances among different frontal lobe disorders, but none of the instruments has proven useful for predicting real-life performance. In the every day life of the patient with a frontal lobe disorder, environmental dependence (the response to action cues from the external environment) may override social judgment [32,33]. Benson [34] describes the case of posttraumatic patient with bifrontal disorder and diabetes insipidus who agreed repeatedly not to drink water and not to approach the water fountain on the hospital ward. Just as many times, he was observed drinking from the same fountain. The patient never forgot the directions but also could not control his actions. This invalidates measures of personality that do not collect data from an informant well-acquainted with the patient.

The Neuropsychiatric Inventory (NPI) [35] and the Behavioral Pathology in Alzheimer's Disease Rating Scale (BEHAVE-AD) [36] are routinely used in evaluation of dementia patients to inventory behavioral disturbances and have been used as outcome measures in clinical drug trials. Both instruments are administered to caregiver informants. When assessing patients with frontal lobe disorders, the NPI may be a more specific and sensitive instrument than the BEHAVE-AD, because the NPI surveys for new onset apathy, changes in appetite (eg, preference for sweets), and hoarding behaviors, which are specific for frontal lobe disorders. The Iowa Rating Scales of Personality Change also collects information from 
a knowledgeable caregiver. It differs from the NPI and the BEHAVE-AD in that it also rates the degree of change between premorbid baseline and after the onset of frontal lobe disorder on 30 personality characteristics [37]. This list of personality characteristics adds inflexibility, poor frustration tolerance, vanity, excitability, indecisiveness, lack of persistence, easily overwhelmed, manipulativeness, and lack of insight to the items found in the NPI and the BEHAVE-AD. All of these instruments rely upon a knowledgeable informant; none of these instruments predicts treatment response or future evolution of personality change.

The Interpersonal Adjective Scale evaluates personality along two major axes: dominance and nurturance. Informants rate patients' premorbid and current personality types by responding to 64 adjectives descriptive of interpersonal interactions ( $\mathrm{eg}$, assertive, ruthless, timid, kind). [38] Premorbid and current personality wedges are then readily comparable on the Likkert scale graph (Fig. 3). Two diametrically opposed wedges indicate the greatest deviation from premorbid personality. Although useful for presenting existing data, this instrument is also unable to predict further personality changes or how long any undesirable behaviors will persist.

The Frontal Lobe Personality Scale can be used to measure premorbid versus current personality characteristics quantitatively [39]. This instrument grants a clinical advantage in that the instrument can be completed by both the patient and a knowledgeable informant in the waiting room, but it can only be completed by relatively intact patients with higher MiniMental State Exam scores, intact language and motor skills. It takes less time for the informant to complete than the Iowa Scales of Personality Change and, unlike caregiverbased instruments, the Frontal Lobe Personality Scale allows the patient to reveal his lack of insight into his change in personality.

The Hare Psychopathy Checklist and its adjunct, the Interpersonal Measure of Psychopathy, are used frequently in forensics to demonstrate the degree to which the individual does not adhere to social norms of interpersonal behaviors [40]. Grandiose sense of self-worth, lack of remorse/guilt, shallow affect, lack of empathy, failure to accept responsibility for own actions, need for stimulation, poor behavioral controls, lack of realistic, long-term plans, impulsivity, and promiscuous sexual behavior are items on the Hare Psychopathy Checklist Revised [41]. The Interpersonal Measure of Psychopathy is an ancillary list of behaviors that might occur during the interview of the subject that would support abnormal Factor 1 scores (affective and interpersonal components of the Psychopathy Checklist). Examples of Interpersonal Measure of Psychopathy items include interrupting the interviewer, ignoring personal boundaries, frustration with argument avoidance, ethical superiority, showmanship, expressive toughness, and intense eye contact [40]. The Hare Psychopathy checklist has been validated for forensic use in criminal offenders to give a prediction of an individual's risk for violent behavior [42]. This instrument was designed for patients with long-standing antisocial personality disorder, which has some important characteristics differentiating it from an orbitofrontal syndrome due to a frontal lobe disorder. Individuals with antisocial personality disorder are quite functional and usually exercise exceptional social skills to manipulate others for their own purposes, without regard for morals adhered to by their 
communities. Items on psychopathy instruments that measure this exploitative ability usually do not apply to patients with acquired frontal lobe disorders.

\section{Conclusions}

Personality changes in frontal lobe disorders may resemble one or any combination of three frontal-subcortical syndromes. Focal frontal lobe disorders can cause more readily predictable changes in personality: orbitofrontal cortex lesions usually lead to a disinhibited, acquired sociopathy; dorsolateral cortex lesions disrupt organization, planning, and judgment; and disruption of anterior cingulate gyri results in apathy. Lesions involving subcortical components of the three frontal-subcortical circuits more frequently lead to combined syndromes due to distribution of the injury over more than one circuit in the caudate, globus pallidus or thalamus. Diffuse insults to frontal cortex lead to changes in personality that can differentiate frontotemporal dementia from AD. Currently available instruments to monitor changes in personality have little application to predicting patient performance in daily living and patient response to treatment.

\section{Acknowledgments}

This work was funded by Alzheimer's Disease Research Center (AG10123) grant from the National Institute on Aging, an Alzheimer's Disease Research Center of California grant, the Sidell-Kagan Foundation, and the Deane Johnson Foundation for Alzheimer's Disease Research.

\section{References and Recommended Reading}

Papers of particular interest, published recently, have been highlighted as:

- Of importance

•• Of major importance

1. American Psychiatric Association. Diagnostic and Statistical Manual of Mental Disorders. American Psychiatric Association; Washington, DC: 1994.

2. Damasio H, Grabowski T, Frank R, et al. The return of Phineas Gage: clues about the brain from a skull of a famous patient. Science. 1994; 264:1102-1105. [PubMed: 8178168]

3. Harlow. Recovery from the passage of an iron bar through the head. Publications of the Massachusetts Medical Society. 1868; 2:329-346.

4. Cummings JL. Frontal-subcortical circuits and human behavior. Arch Neurol. 1993; 50:873-880. [PubMed: 8352676]

5. Tranel D. "Acquired sociopathy": the development of sociopathic behavior following focal brain damage. Prog Exp Pers Psychopathol Res. 1994:285-311. [PubMed: 8044207]

6. Zahn TP, Grafman J, Tranel D. Frontal lobe lesions and electrodermal activity: effects of significance. Neuropsychologia. 1999; 37:1227-1241. [PubMed: 10530723]

7••. Chow, TW., Cummings, JL. Frontal-subcortical circuits. In: Miller, BL., Cummings, JL., editors. The Human Frontal Lobes. Functions and Disorders. Guilford Press; London: 1999. p. 3-26.This textbook contains a few chapters cited in this review and is the most current reference dedicated to frontal lobe function

8. Mega MS, Cummings JL. Frontal-subcortical circuits and neuropsychiatric disorders [see comments]. J Neuropsychiatry Clin Neurosci. 1994; 6:358-370. [PubMed: 7841807]

9••. Schnider, A., Gutbrod, K. Traumatic brain injury. In: Miller, BL., Cummings, JL., editors. The Human Frontal Lobes. Functions and Disorders. Guilford Press; London: 1999. p. 487-506.An excellent reference 
10. Benjamin S, Kirsch D, Visscher T, et al. Hypomania from left frontal AVM resection. Neurology. 2000; 54:1389-1390. [PubMed: 10746620]

11. Baxter LR. Brain imaging as a tool in establishing a theory of brain pathology in obsessivecompulsive disorder. J Clin Psychiatry. 1990; 51:22-26.

12. Greenberg BD, Ziemann U, Cora-Locatelli G, et al. Altered cortical excitability in obsessivecompulsive disorder. Neurology. 2000; 54:142-147. [PubMed: 10636140]

13. Garvey MA, Giedd J, Swedo SE. PANDAS: the search for environmental triggers of pediatric neuropsychiatric disorders. Lessons from rheumatic fever. J Child Neurol. 1998; 13:413-423. [PubMed: 9733286]

14. Kurlan R. Tourette's syndrome and "PANDAS": will the relation bear out? Pediatric autoimmune neuropsychiatric disorders associated with streptococcal infection. Neurology. 1998; 50:15301534. [PubMed: 9633690]

15•. Swedo SE, Leonard HL, Garvey M, et al. Pediatric autoimmune neuropsychiatric disorders associated with streptococcal infections: clinical description of the first 50 cases. Am J Psychiatry. 1998; 155:264-271. Primary source for descriptions of cases of pediatric auto immune neuropsychiatric disorders associated with streptococcal infection. [PubMed: 9464208]

16. Mimura M, White RF, Albert ML. Corticobasal degeneration: neuropsychological and clinical correlates. J Neuropsychiatry Clin Neurosci. 1997; 9:94-98. [PubMed: 9017535]

17. Schneider JA, Watts RL, Gearing M, et al. Corticobasal degeneration: neuropathologic and clinical heterogeneity. Neurology. 1997; 48:959-969. [PubMed: 9109885]

18. Costa PTJ, McCrae RR. Personality in adulthood: a six-year longitudinal study of self-reports and spouse ratings on the NEO Personality Inventory. J Pers Soc Psychol. 1988; 54:853-863. [PubMed: 3379583]

19. Levy M, Miller BL, Cummings JL, et al. Alzheimer's disease and frontotemporal dementias: behavioral distinctions. Arch Neurol. 1996; 53:687-690. [PubMed: 8929178]

20. Mendez MF, Perryman KM, Miller BL, Cummings JL. Behavioral differences between frontotemporal dementia and Alzheimer's disease: a comparison on the BEHAVE-AD rating scale. Int Psychogeriatrics. 1998; 10:155-162.

21. Mychack P. Novel applications of social-personality measures to the study of dementia. Neurocase. 2000 in press.

22. Swartz R, Miller BL, Lesser IM, et al. Behavioral phenomenology in Alzheimer's disease, frontotemporal dementia, and late-life depression: a retrospective analysis. J Geriatr Psychiatry Neurol. 1997; 10:67-74. [PubMed: 9188022]

23. Mega MS, Masterman DM, O'Connor SM, et al. The spectrum of behavioral responses to cholinesterase inhibitor therapy in Alzheimer disease. Arch Neurol. 1999; 56:1388-1393. [PubMed: 10555660]

24. Mychack P. The influence of right frontotemporal dysfunction on social behavior in frontotemporal dementia. Neurology. 2000 in press.

25. Chu CC, Tranel D, Damasio AR, Van Hoesen GW. The autonomic-related cortex: pathology in Alzheimer's disease. Cerebral Cortex. 1997; 7:86-95. [PubMed: 9023436]

26. Chu CC, Tranel D, Damasio AR. Impaired autonomic responses to emotionally significant stimuli in Alzheimer's disease. Society of Neuroscience Abstracts. 1994; 20:1006.

27. Hirono N, Mori E, Ishii K, et al. Frontal lobe hypometabolism and depression in Alzheimer's disease. Neurology. 1998; 50:380-383. [PubMed: 9484357]

28••. McKeith, IG. Lewy body disorders. In: Miller, BL., Cummings, JL., editors. The Human Frontal Lobes. Functions and Disorders. Guilford Press; London: 1999. p. 422-435.An excellent reference

29•. Hirono N, Mori E, Tanimukai S, Kazui H, Hashimoto M, Hanihara T, et al. Distinctive neurobehavioral features among neurodegenerative dementias. J Neuropsychiatry Clin Neurosci. 1999; 11:498-503. Reviews many dementias and neuropsychiatric symptoms associated with them. [PubMed: 10570764]

30. Lopez-Rodriguez F, Altshuler L, Kay J, Delarhim S, et al. Personality disorders among refractory epileptic patients. J Neuropsychiatry Clin Neurosci. 1999; 11:464-469. [PubMed: 10570759] 
31. Anderson SW, Bechara A, Damasio H, et al. Impairment of social and moral behavior related to early damage in human prefrontal cortex. Nat Neurosci. 1999; 2:1032-1037. [PubMed: 10526345]

32. Lhermitte F. Human autonomy and the frontal lobes. Part II. Patient behavior in complex and social situations: the "environmental dependency syndrome". Ann Neurol. 1986; 19:335-343. [PubMed: 3707085]

33. Lhermitte F. "Utilization" behavior and its relation to lesions of the frontal lobes. Brain. 1983; 106:237-255. [PubMed: 6850269]

34. Benson, DF. The Neurology of Thinking. Oxford University Press; New York: 1994.

35. Cummings JL, Mega M, Gray K, et al. The Neuropsychiatric Inventory: comprehensive assessment of psychopathology in dementia. Neurology. 1994; 44:2308-2314. [PubMed: 7991117]

36. Reisberg B, Borenstein J, Salob SP, Ferris, et al. Behavioral symptoms in Alzheimer's disease: phenomenology and treatment. J Clin Psychiatry. 1987; 48:9-15.

37. Wiggins JS, Trapnell P, Phillips N. Psychometric and geometric characteristics of the revised Interpersonal Adjective Scales (IAS-R). Multivariate Behav Res. 1988; 23:517-530. [PubMed: 26761163]

38. Grace J, Stout JC, Malloy PF. Assessing frontal behavioral syndromes with the Frontal Lobe Personality Scale. Assessment. 1999; 6:269-284. [PubMed: 10445964]

39. Kosson DS, Steurerwald BL, Forth AD, Kirkhart KJ. A new method for assessing the interpersonal behavior of psychopathic individuals: preliminary validation studies. Psychol Assessment. 1997; 9:89-101.

40. Hare, RD. The Hare Psychopathy Checklist—Revised. Multi-Health Systems; Toronto: 1991.

41. Salekin RT, Rogers R, Sewell KW. A review and meta-analysis of the Psychopathy Checklist and Psychopathy Checklist-Revised: predictive validity of dangerousness. Clin Psychol Sci Pract. 1996; 3:203-215.

42. Stuss, DT., Benson, DF. The Frontal Lobes. Raven Press; New York: 1986. 


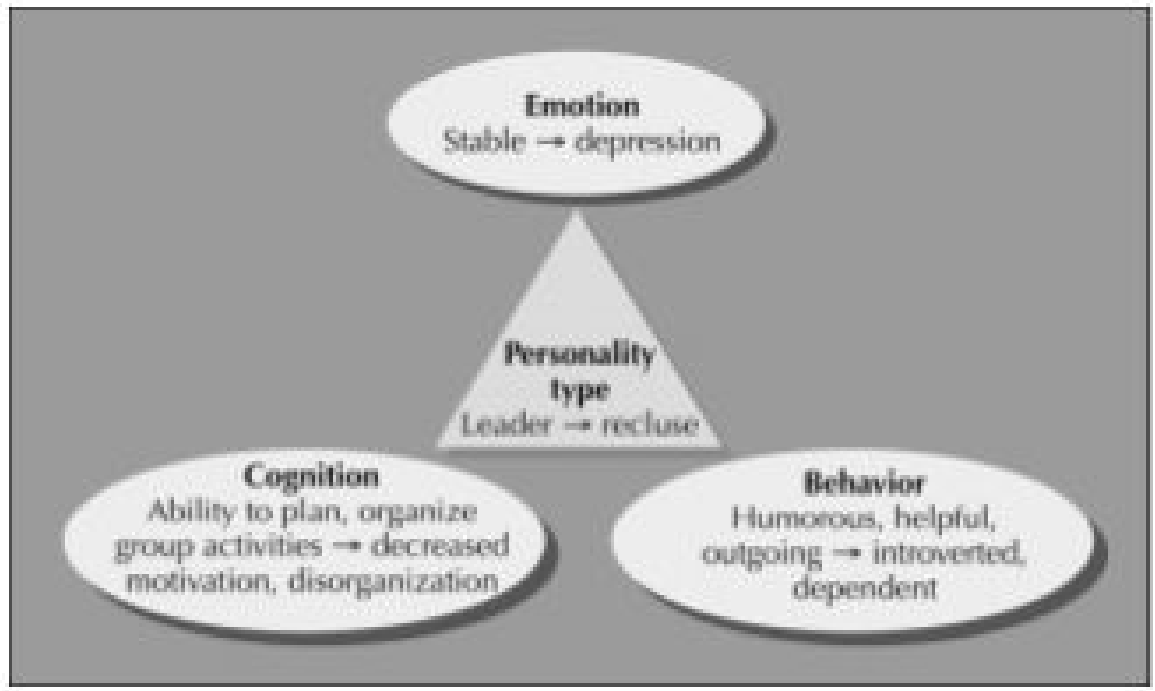

Figure 1.

One example of how emotional, cognitive, and behavioral changes compose an acquired change in personality. 


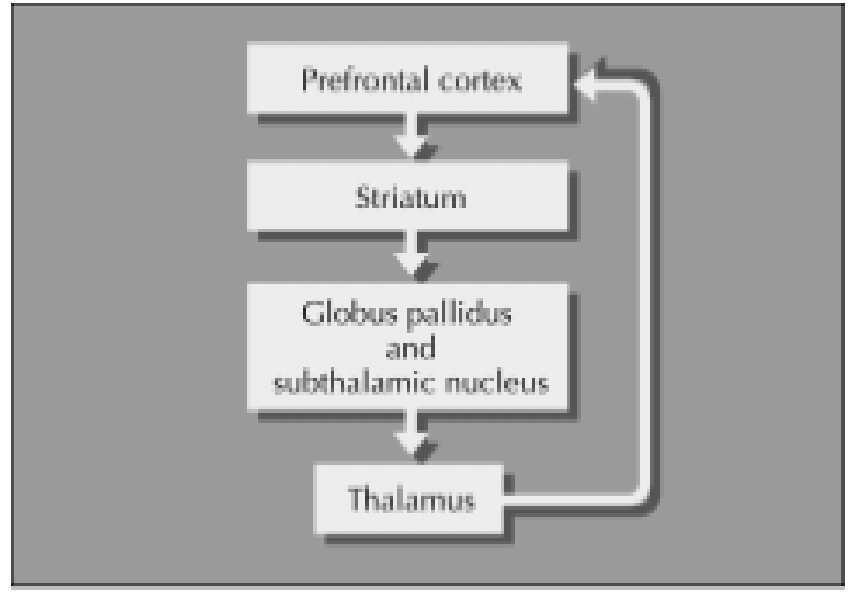

Figure 2.

The frontal-subcortical circuit model. 


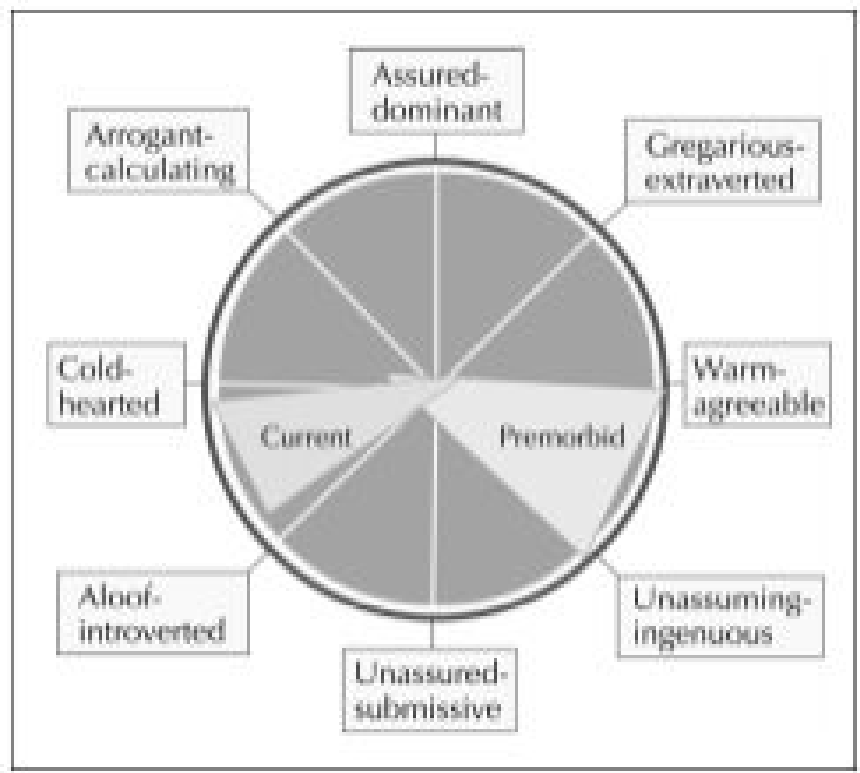

Figure 3 .

Example of Interpersonal Adjective Scale in a patient with frontotemporal dementia. (Adapted from Grace et al. [38].) 
Table 1

Characteristics of the orbitofrontal-subcortical circuit syndrome

Blunted emotional experience or emotional dysregulation

Impaired judgment

Indecisiveness

Lack of persistence

Lack of planning

Lack of insight into acquired changes

Social inappropriateness: tactlessness, facetiousness, puerile attitude 


\section{Table 2}

Frontal lobe disorders that can cause personality changes

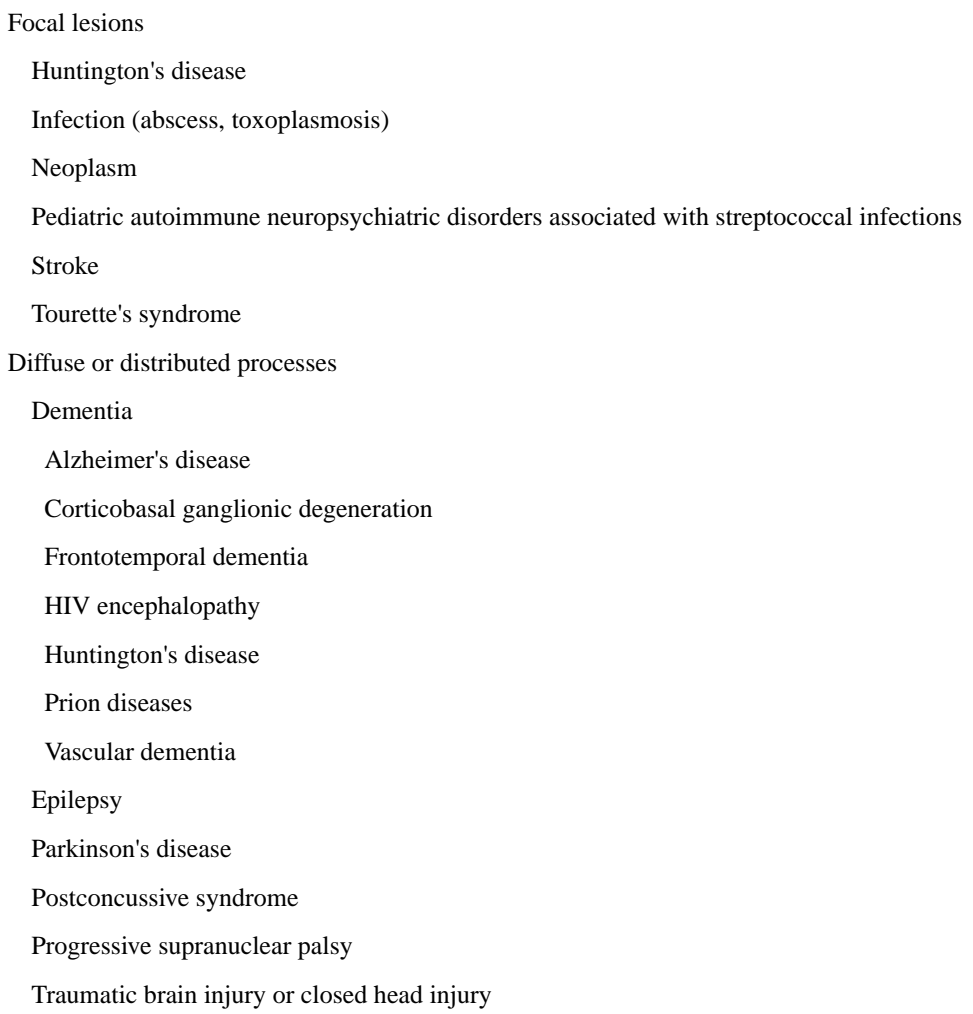

